\title{
A Genetic Murine Model of Endolymphatic Hydrops: The Phex Mouse
}

\author{
Cameron C. Wick • Maroun T. Semaan • \\ Qing Yin Zheng $\cdot$ Cliff A. Megerian
}

Published online: 24 May 2014

(c) Springer Science+Business Media New York 2014

\begin{abstract}
Animal models of endolymphatic hydrops (ELH) provide critical insight into the pathophysiology of Meniere's disease (MD). A new genetic murine model, called the Phex mouse, circumvents prior need for a time and cost-intensive surgical procedure to create ELH. The Phex mouse model of ELH, which also has X-linked hypophosphatemic rickets, creates a postnatal, spontaneous, and progressive ELH whose phenotype has a predictable decline of vestibular and hearing function reminiscent of human MD. The Phex mouse enables realtime histopathologic analysis to assess diagnostic and therapeutic interventions as well as further our understanding of ELH's adverse effects. Already the model has validated electrocochleography and cervical vestibular evoked myogenic potential as useful diagnostic tools. New data on caspase activity in apoptosis of the spiral ganglion neurons may help target future therapeutic interventions. This paper highlights the development of the Phex mouse model and highlights its role in characterizing ELH.
\end{abstract}

C. C. Wick · M. T. Semaan - Q. Y. Zheng ·

C. A. Megerian ( $\square)$

Ear, Nose, and Throat Institute, University Hospitals Case

Medical Center, Case Western Reserve University, 11100 Euclid

Avenue, Cleveland, OH 44106, USA

e-mail: cliff.megerian@uhhospitals.org

C. C. Wick

e-mail: cameron.wick@uhhospitals.org

M. T. Semaan

e-mail: maroun.semaan@uhhospitals.org

Q. Y. Zheng

e-mail: qyz@case.edu
Keywords Phex $\cdot$ Endolymphatic hydrops - Meniere's disease $\cdot$ Electrocochleography $\cdot$ Vestibular evoked myogenic potential $\cdot$ Apoptosis

\section{Introduction}

The combination of tinnitus, fluctuating hearing loss, vertigo, and aural fullness was first described by Prosper Ménière in 1861 [1]. Later termed as Meniere's disease (MD), the underlying pathophysiology of these symptoms was not elucidated until 1938 when two nearly simultaneous independent posthumous reports linked MD to endolymphatic hydrops (ELH) [2, 3]. Endolymphatic hydrops is defined as increased fluid volume within the endolymphatic space causing distention of Reissner's membrane, and its association with MD has been supported by numerous investigators [4-8].

ELH is not exclusive to MD. Secondary ELH causing Ménière-like symptoms has been described with endolymphatic sac tumors [9], meningiomas [10], acoustic neuromas [11], and relapsing polychondritis [12]. The American Academy of Otolaryngology-Head and Neck Surgery defines MD as "an idiopathic syndrome of endolymphatic hydrops" thereby distinguishing it from secondary ELH [13].

Despite the well-established role of ELH in MD, our current knowledge of why ELH develops, how it produces symptoms, and our ability to predict disease progression or treatment response remains rudimentary. While efforts have been made to diagnose ELH via three-dimensional fluid-attenuated inversion recovery (3D-FLAIR), magnetic resonance imaging (MRI) [8, 14, 15], and electrocochleography (ECochG) [16], the gold standard remains posthumous histopathology. Since appropriate human temporal bone specimens are difficult to obtain, animal models of 
ELH provide a critical tool for researchers to better understand the pathophysiology and sequelae of ELH.

\section{Previous Animal Models of Endolymphatic Hydrops}

The guinea pig served as the first animal model for ELH. In 1965, Kimura and Schuknecht were able to create secondary ELH in a guinea pig via surgical obliteration of the endolymphatic duct (ED) $[17,18]$. The original intradural technique was later modified to an extradural approach, but still associated with significant surgical morbidity and intensive animal care $[19,20]$. Limitations of this model included production of secondary ELH that is inherently different than the spontaneous ELH of MD. Additionally, there was an inconsistent production of vestibular symptoms [21].

Despite these limitations, the surgical guinea pig model has provided valuable insight into the pathophysiology of ELH. Fundamental principles of ELH garnered from this model include the development of ELH at the cochlea apex, progression of the hydrops throughout the inner ear, and initial low-frequency sensorineural hearing loss (SNHL) that evolves into a profound SNHL across all frequencies [18, 22, 23]. Neurotoxicity, whether initiated by pressure or biochemical means, is known to play a critical role in the pathophysiology of MD. Through the guinea pig model, the sequelae of neural damage and SNHL have begun to take shape. Spiral ganglion neurons (SGNs) degenerate in an apical to basal pattern [24], which precedes the degeneration of inner hair cells [25] and ultimately results in a reduced cochlear nerve diameter [26]. The relationship between the degree of ELH and the degree of SNHL was correlated in humans [27] and recapitulated in the guinea pig model $[28,26]$.

A less invasive attempt to produce ELH in the guinea pig involves injection of an artificial endolymph into the endolymphatic space. The physiologic effect of this hydrops is dependent upon the volume and rate of the injected material. This technique is susceptible to membrane rupture, and the desired hydropic effect is only temporary. Efforts to prolong the duration of injectable ELH have utilized hyaluronate gel, but this technique has had limited research application [29]. For these reasons, it was desirable to find an animal model that more closely replicates the spontaneous ELH, hearing loss, and vestibular symptomatology of human MD.

\section{Development of the Phex Murine Model for Endolymphatic Hydrops}

Genetic mouse models are commonly used to investigate human disease. Originally, two mouse models existed for the study of X-linked hypophosphatemic rickets, the most common form of heritable rickets. These models, referred to as Phex ${ }^{\text {Hyp }}$ (Hyp) and Phex ${ }^{G y}$ (Gy), both carry genetic defects that result in loss-of-function of the phosphate-regulating gene with homology to endopeptidases located on the $\mathrm{X}$ chromosome (PHEX) [30, 31]. The PHEX gene encodes the PHEX protein, which is a zinc-metalloendopeptidase located in the cell membrane of osteoblasts and chondrocytes [32]. The protein's normal function is to bind pro-mineralization factors, like dentin matrix protein-1 (DMP-1) and matrix extracellular phosphoglycoprotein (MEPE), to support healthy bone mineralization. A defective PHEX protein leads to unmineralized osteoid deposition and soft bone, termed osteomalacia and rickets. Aditionally, the impaired PHEX-MEPE binding causes an increase in fibroblast growth factor-23 (FGF-23) resulting in renal phosphate wasting and decreased 1,25-dihydroxyvitamin D production [33, 34].

Both the Hyp and Gy models exhibit the hallmarks of $\mathrm{X}$-linked hypophosphatemic rickets such as hypophosphatemia caused by renal phosphate wasting, osteomalacia, mild hypocalcemia, and shortened limbs. Observation of the male Gy mouse also showed hyperactivity, inner ear abnormalities, and progressive circling behavior [35]. Unfortunately, male Gy mice are sterile and have reduced viability, thus limiting their usefulness as a research tool. The manifestation of otologic problems in the Gy mouse has precedence, as many correlations between X-linked hypophosphatemia and ear abnormalities have been described in humans. These specifically include SNHL, thickening of the petrous temporal bone, narrowing of the internal auditory canal, and ELH confirmed by ECochG [36-41].

In 2004, Lorenz-Depiereux et al. [42] described a new mouse strain called Phex ${ }^{\text {Hyp-Duk }}$ (Hyp-Duk). The Hyp-Duk mouse arose from a spontaneous loss-of-function mutation in the Phex gene. The genetic defect is isolated to the Phex gene and is bred in mice with a BALB/cAnBomUrd-Foxn $1^{n u}$ (BALB/cUrd) background. Importantly, they observed that male Hyp-Duk (Hyp-Duk/Y) mice exhibited circling behavior indicative of vestibular dysfunction, in addition to enlargement of the scala media and hearing loss.

\section{Otologic Manifestations in the Phex Murine Model}

In 2008, Megerian et al. [43*0] recognized the unique attributes of the Hyp-Duk/Y strain and sought to further characterize the inner ear phenotype of this new model. They found that, in addition to the previously described morphologic features of $\mathrm{X}$-linked hypophosphatemic rickets, the Hyp-Duk/Y reliably developed progressive postnatal signs of inner ear dysfunction. Vestibular sequelae, including circling behavior, head bobbing, and/or unstable gait typically began by postnatal day (P) 15 and were obvious by $\mathrm{P} 30$. 
The early decline in the Hyp-Duk/Y vestibular function led Megerian et al. to test hearing at a younger age that had previously been reported. Using a combination of click and toneburst stimuli, auditory brainstem response (ABR) testing was used to evaluate hearing function at multiple time points between P21 (weaning) and P131. At P21, most mutants showed near-normal hearing levels in at least one ear. By P25 mutants demonstrated varying degrees of bilateral hearing loss with a proclivity for low-frequency loss to precede highfrequency loss. Hearing fluctuation stabilized with time and in some litters progressed to profound hearing loss.

With evidence of spontaneous, progressive, postnatal cochleovestibular dysfunction in Hyp-Duk/Y mice, Megerian et al. correlated these phenotypic changes with inner ear histology. At birth the Hyp-Duk/Y inner ear was identical to agematched controls, including no evidence of ELH, spiral ganglion degeneration, or ED obstruction. Apical hydrops became apparent by P25, along with thickening and osteomalacia of the surrounding otic capsule bone. By P40 all mutants demonstrated significant ELH, yet SGNs and hair cells remained largely intact. By P90, a time point in which the Hyp-Duk/Y typically has profound hearing loss, severe hydrops had progressed throughout the cochlea and vestibule. Significant SGN degeneration was present at all cochlear turns but most pronounced at the apical turn. The ED remained patent and the hair cells were mostly preserved [43••]. Figure 1 illustrates the postnatal time course and phenotypic changes described above (Fig. 1).

\section{Characterization of Spiral Ganglion Neuron Death in Endolymphatic Hydrops}

Data from Hyp-Duk/Y mice demonstrate that functional cochleovestibular deficits occur prior to inner ear structural

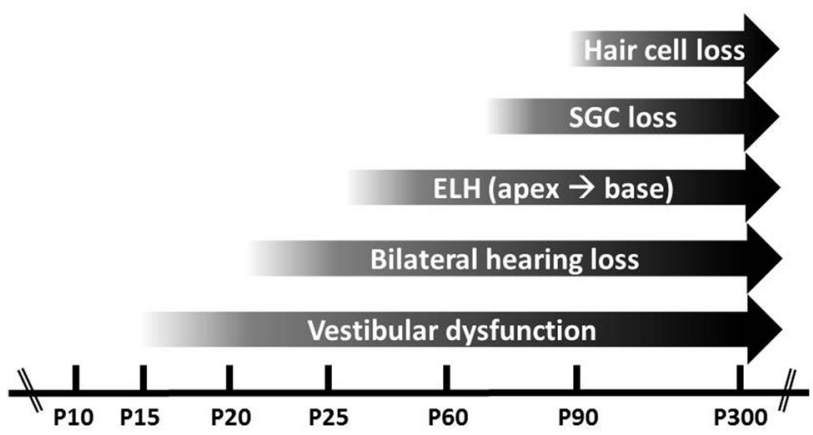

Fig. 1 Phenotypic development of Phex ${ }^{\text {Hyp-Duk }}$ (Hyp-Duk/Y) mice. Phex ${ }^{\text {Hyp-Duk }}$ (Hyp-Duk/Y) mice develop a characteristic postnatal (P) phenotype that includes vestibular dysfunction (head bobbing, circling behavior), hearing loss, endolymphatic hydrops (ELH), spiral ganglion neuron (SGN) loss, and hair cell loss. Faded portion of the lines indicates variable onset of the phenotype deterioration. Both the Hyp-Duk/Y and guinea pig surgical models support the theory of ELH progression from the cochlear apex to the cochlear basal turn. The animal models also agree that SGN degeneration progresses in an orientation similar to ELH and precedes hair cell loss [24, 25]. Human temporal bone specimens in patients with MD recapitulate these experimental findings [44-46].

While the topographic pattern of SGN degeneration in ELH has been well-established, quantitative and mechanistic data were lacking until Semaan et al. (2013) isolated caspase activity within in the spiral ganglia of Hyp-Duk/Y mice $[47 \bullet \bullet$. Caspases are a family of cysteine proteases that activate the apoptotic cascade [48]. Caspase-3, caspase-8, and caspase-9 activity in the Hyp-Duk/Y and control mice were analyzed with immunohistochemical stains, semi-quantitative reverse transcriptase-polymerase chain reaction (RT-PCR), quantitative PCR, and terminal deoxynucleotidyl transferase Y-mediated dUTP-biotin nick-end labeling assay (TUNEL). Mutants and controls were analyzed from P21 to P120. All the methods of measurement indicated some degree of increased caspase activity in the Hyp-Duk/Y mice compared to controls. Caspase was strongly expressed in the spiral ganglia of Hyp-Duk/Y mice, and both its apical to basal topographic distribution and time course foreshadowed the SGN cell death (Fig. 2). Interestingly, by P90 there was no evidence of significant caspase activity in the Organ of Corti, which suggests that eventual hair cell loss in ELH may not be an apoptotic event, but rather loss of neurotrophic factors as afferent dendrites of SGNs degenerate [47••, 49].

Quantitative analysis of SGN density at P60, P90, and P120 quantified the long-observed chronologic progression of SGN loss associated with ELH. At P60, there was no difference in SGN populations between Hyp-Duk/Y mutants and controls. By P90 and P120, a sizeable decline of SGN density at the apical turn of mutant mice had developed ( $p=0.003$ and $p=0.0002$, respectively) [47••]. This study definitively indicates apoptosis as a critical element in the SGN degeneration associated with ELH.

\section{Diagnostic Implications}

The diagnosis of MD remains a challenging task as it incorporates specific clinical criteria and excludes other causes that can mimic the disorder [13]. Clinical history and audiometry remain the most important diagnostic features. Adjunctive tests for the diagnosis of MD include electrophysiologic studies like ECochG and cervical vestibular evoked myogenic potential (cVEMP), vestibular tests like videonystagmography (VNG) with or without caloric testing and head-thrust testing, and retrocochlear studies like ABR and MRI [50॰]. The use of these 

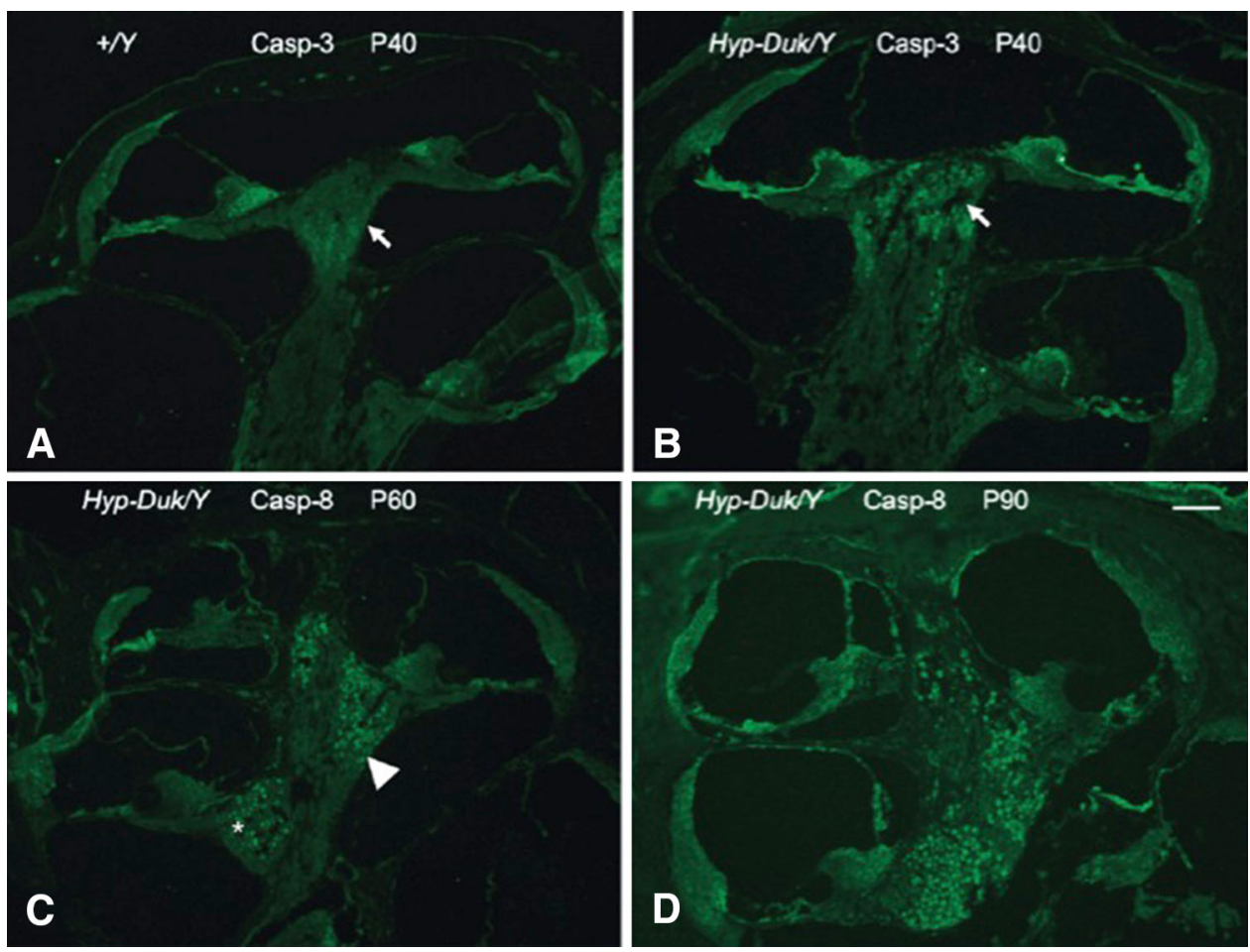

Fig. 2 Topographic distribution of caspase expression in the Phex ${ }^{\text {hyp- }}$ $\mathrm{duk} / \mathrm{Y}$ mutant. All panels are low-power photomicrographs of an immunostained mid-modiolar cochlear section. Scale bar (applies to all panels) $=100 \mu \mathrm{m}$. a Depicts a control mouse at P40 without any significant caspase expression. The white arrow indicates the spiral ganglion neuron (SGN) population at the cochlea apex. b Caspase-3 expression is increased at the apex of a Phex ${ }^{\text {hyp-duk/Y }}$ mutant at P40. No significant expression is seen in the middle and basal turns. c Caspase- 8 expression is increased in all turns of the cochlea of a

adjunctive tests varies widely among otolaryngologists [51].

The first use of ECochG in a human is accredited to Ruben et al. in 1960, but it was not until 1974 when Schmidt et al. first applied it as a diagnostic tool for MD $[52,53]$. ECochG records evoked potentials from the cochlea in response to a sound stimulus. Either intratympanic or extratympanic surface electrodes measure the evoked potentials, which create a waveform of the electrical events within the cochlea. ECochG has three components: the cochlear microphonic $(\mathrm{CM})$ is an alternating current that reflects the instantaneous displacement of the basilar membrane; the summating potential (SP) reflects hair cell response to a sound stimulus; and the action potential (AP) represents the collective firing of auditory nerve fibers $[54,55]$. An elevated SP/AP ratio $(>0.4)$ and/ or a widened AP width $(>3 \mathrm{~ms})$ is considered diagnostic for ELH. The elevated SP/AP ratio is hypothesized to reflect a mechanical asymmetry of the basilar membrane [56]. While many clinical studies support the relationship between an elevated SP/AP ratio and ELH, recently there
Phex ${ }^{\text {hyp-duk/Y }}$ mutant at P60. The white arrowhead and asterisk indicate SGN population of the middle and basal turns, respectively. d Caspase- 8 expression is increased at the basal turn of the cochlea in a Phex ${ }^{\text {hyp-duk/Y }}$ mutant at P90. Note the progression of caspase expression from the cochlea apex to the basal turn from P40 to P90 (panels b, c, and d). Figure originally published as Semaan MT, et al. in Otology and Neurotology (2013;34:559-569) Permission for republication granted from Wolters Kluwer Health

had been no in vivo confirmation [57, 58]. Furthermore, all previous reports of ECochG in animals used the surgical guinea pig model with an obstructed ED that conceivably has different endolymphatic fluid mechanics than the spontaneous ELH of MD [59].

The Hyp-Duk/Y model presents an opportunity to measure electrophysiologic data and instantly correlate it with inner ear histopathology. This was the approach taken by Melki et al. (2014) who measured 41 control ears and 25 Hyp-Duk/Y ears with ABR and ECochG after which they performed a real-time histopathologic analysis of ELH. They found that an elevated SP/AP ratio predicted the presence of ELH in Hyp-Duk/Y mice (mean SP/AP ratio in Hyp-Duk/Y was 0.44 compared to 0.28 in controls, $p=0.0003$ ) $\left[60^{\bullet}\right]$. They did not find a linear relationship between the SP/AP ratio and the severity of ELH or the hearing threshold. However, they did note a relationship between the hearing threshold and the degree of ELH $(p<0.0002)$. Data analysis with a receiver operating curve (ROC) confirmed ECochG's ability to correctly diagnose the hydropic state and the calculated sensitivity (58\%), 
Fig. 3 Electrocochleography waveforms from control and Phex ${ }^{\text {hyp-duk/Y }}$ mutant. Sample electrocochleography (ECochG) waveforms from a control and Phex ${ }^{\text {hyp-duk/Y }}$ mutant. The baseline (B), summating potential (SP), and action potential (AP) are indicated for each genotype. In this sample, the SP/AP ratios are 0.2 and 0.5 for the control and mutant mice, respectively. Figure originally published by Melki SJ, et al. in Journal of the Association for Research in Otolaryngology (2014, epub ahead of print; doi: 10.1007/s10162-014-0445-0). Permission for republication granted from Springer

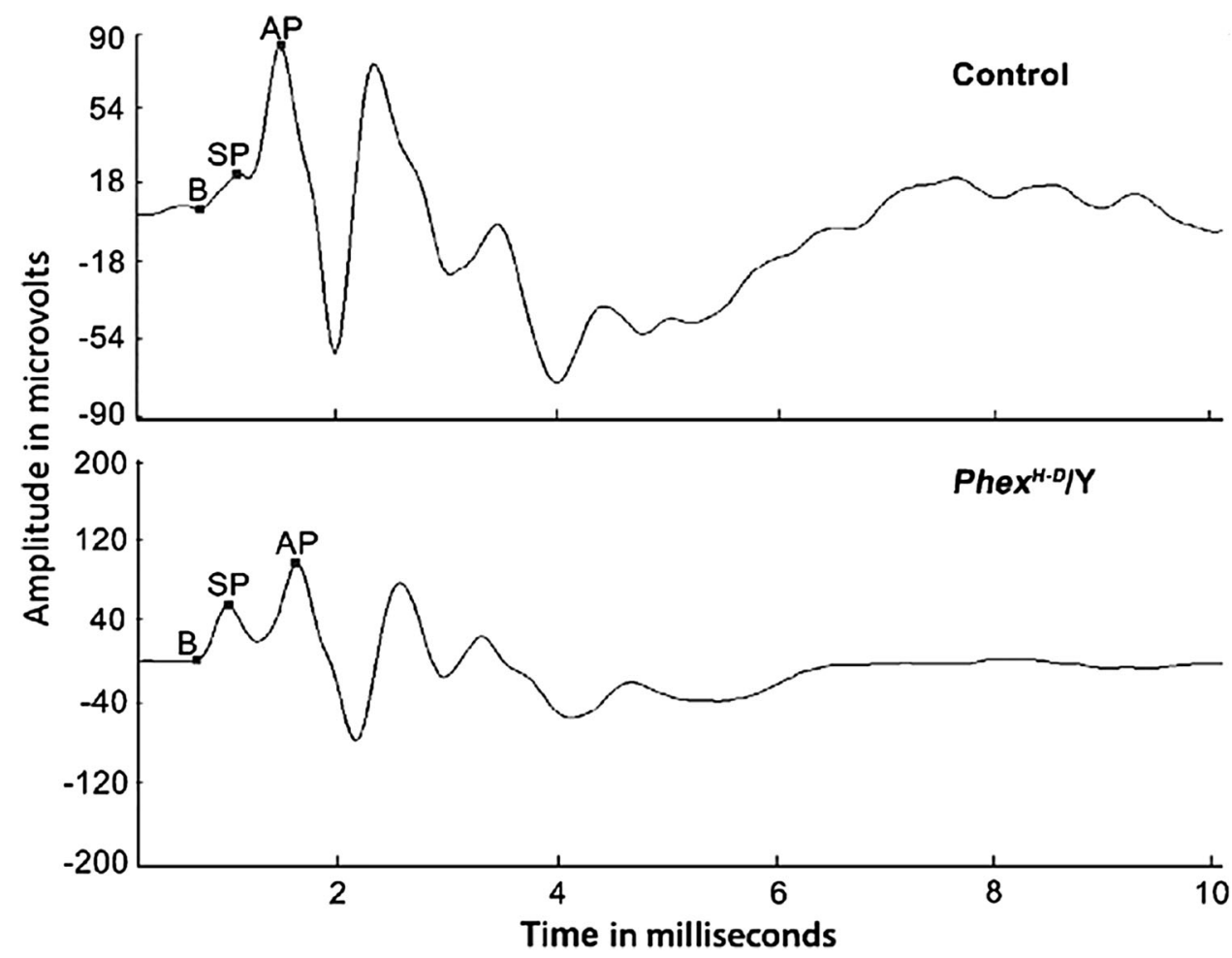

specificity (93\%), positive predictive value (83\%), and negative predictive value $(78 \%)$ are consistent with ECochG data from human patients with MD (Fig. 3) [58, 60•]. Melki et al. also provide a minimally invasive (extratympanic) ECochG method that should facilitate future longitudinal studies.

The cervical VEMP (cVEMP) is a short latency inhibitory potential of the ipsilateral sternocleidomastoid muscle evoked by a brief and loud $(>85 \mathrm{~dB})$ monaural click or tone-burst stimulus. The cVEMP is believed to be a recording of the vestibulocollic reflex generated in the saccule and carried via the inferior vestibular nerve. Human studies of cVEMP indicated elevated or absent thresholds in patients with MD [61]. In 2009, Sheykholeslami et al. [62] first reported a method to perform cVEMP on Hyp-Duk/Y mice. Similar to Melki et al. (2014), they correlated the electrophysiologic data, in this case cVEMP and hearing thresholds, with real-time histopathologic analysis. Ten adult (P60-P90) control mice had a cVEMP threshold of $60 \mathrm{~dB}$ normal hearing level (nHL), while five age-matched Hyp-Duk/Y mice had either absent or elevated (100 dB nHL) cVEMP thresholds.

\section{Therapeutic Implications}

Therapeutic options for patients with MD range from a conservative low-salt diet to complete ablation of labyrinthine function. Clinicians' inability to predict therapeutic response adds to both patient and clinician frustration with MD management [50•, 63].

Extrapolating data from the Hyp-Duk/Y model to human patients with MD has translational potential. Semaan et al. (2013) proved that caspase activation and the apoptotic cascade are the final steps before SGN degeneration $[47 \bullet \bullet$. It remains unknown what initiates that apoptotic cascade. Theories of pressure from hydropic ear, ionic perturbations, and excitotoxicity have all been proposed.

To test the theory of excitotoxicity and the role of glutamate-activated reactive oxygen species (ROS) in earlystage hearing loss caused by ELH, Melki et al. (2010) systemically injected riluzole (a glutamate release inhibitor) plus dimethylsulfoxide (DMSO; an anti-inflammatory and anti-oxidant solvent) or DMSO alone in Hyp-Duk/Y mice daily from P6 to P30. Their data show a significant improvement of cochlear function index in both experimental drug groups compared to untreated Hyp-Duk/Y mice at $\mathrm{P} 21$ and $\mathrm{P} 25$ ( $p<0.001$ in both, respectively). There was no synergistic effect of adding riluzole to DMSO, compared to DMSO alone [64]. This study supports the theory of excitotoxicity and/or ROS as a step in ELH-associated hearing loss and demonstrates potential for oto-protective drug therapy. Other drug therapies, like short interfering ribonucleic acid (siRNA) have also shown oto-protective qualities in cisplatin and aminoglycoside ototoxic models and maybe applicable to ELH-associated hearing loss $[65,66]$. Neuroprotective qualities of siRNA therapy targeting caspase-induced apoptosis have already 
proven to be efficacious in a rat model of optic nerve damage [67]. Early data using the Hyp-Duk/Y model suggests siRNA therapy against caspase- 3 preserves SGNs and vestibular ganglion neurons from ELH-associated hearing loss (unpublished data from Zheng QY, Feinstein E, and Megerian CA).

Treatment for humans with X-linked hypophosphatemia rickets centers on phosphate repletion and calcitriol administration [68]. This has demonstrated improvement in bone mineralization and growth, although it elevates the downstream molecule, FGF-23, that is directly responsible for renal phosphate wasting and poor mineralization [69, 70]. The Hyp-Duk/Y mice also suffer from osteomalacia, including at the otic capsule [43••]. Treating Hyp-Duk/Y mice with phosphate and calcitriol does improve otic capsule mineralization but appears to have a limited effect on ELH and hearing threshold (unpublished data from Wick CC, Zheng QY, Megerian CA). Future studies in humans and animals may find further improvement in bone mineralization with anti-FGF23 therapy, but the effect on ELH is unknown [71].

\section{Conclusion}

Animal models of ELH have enabled a basic understanding of the complex pathophysiologic sequelae behind ELH. Early guinea pig models were limited by a time-intensive and delicate surgical operation that resulted in a secondary ELH that was inherently different from the ELH that is seen in patients with MD, and produced variable cochleovestibular dysfunction. Identification of inner ear dysfunction amidst some $\mathrm{X}$-linked hypophosphatemic mice set the stage for the Megerian et al. (2008) description of the Phex murine model (Hyp-Duk/Y). This model serves as an attractive research tool for the study of ELH because it does not require surgical intervention and creates an ear phenotype reminiscent of the cochleovestibular dysfunction of patients with MD.

While the PHEX mutation is not directly responsible for ELH development, the Phex murine model (Hyp-Duk/Y) does provide an easily reproducible, viable mouse that develops spontaneous, progressive, postnatal asymmetric hearing loss and vestibular dysfunction. Much insight into ELH has already been gained from the Phex model, for instance confirmation of ELH and SGN degeneration in an apical to basal manner and functional decline preceding inner ear structural changes. Unlike some other models of hearing loss, ELH causes SGN loss prior to hair cell deterioration.

The past few years have seen a steady increase in the utilization of the Phex mouse (Hyp-Duk/Y) for ELH research. Elegant histologic, physiologic, and molecular studies have expanded the knowledge of ELH pathophysiology. New insight into the role of apoptosis in SGN degeneration, real-time histopathologic confirmation of diagnostic modalities, and future neuroprotective therapies will enable the Phex mouse to be a key contributory to translational research for MD.

Acknowledgments This work was supported in part by a grant from the American Otologic Society to Dr Cliff A. Megerian, and by the NIH grants R01DC007392, R01DC009246 to Dr Qing Y. Zheng.

Compliance with Ethics Guidelines

Conflict of Interest Cameron C. Wick, Maroun T. Semaan, Qing Yin Zheng, and Cliff A. Megerian declare that they have no conflict of interest.

Human and Animal Rights and Informed Consent This article does not contain any studies with human or animal subjects performed by any of the authors.

\section{References}

Recently published papers of particular importance have been highlighted as:

- Of importance

•• Of major importance

1. Ménière P. Maladies de l'oreille interne offrant les symptomes de la congestion cérébrale apoplectiforme. Gaz Méd de Paris. 1861;16:88.

2. Hallpike CS, Cairns H. Observations on the pathology of Meniere's syndrome. J Laryngol Otol. 1938;53:625-55.

3. Yamakawa K. Uber die pathologische Veranderung bei einem Meniere-Kranken. J Otorhinolaryngol Soc Jpn. 1938;44:2310-2.

4. Arenberg IK, Marovitz WF, Shambaugh GE Jr. The role of the endolymphatic sac in the pathogenesis of endolymphatic hydrops in man. Acta Otolaryngol. 1970;275(Suppl):1-49.

5. Schuknecht HF. Pathophysiology of endolymphatic hydrops. Arch Otorhinolaryngol. 1976;212:253-62.

6. Paparella MM. Pathogenesis of Meniere's disease and Meniere's syndrome. Acta Otolaryngol. 1984;406(Suppl):10-25.

7. Merchant SN, Adams JC, Nadol JB Jr. Pathophysiology of Meniere's syndrome: are symptoms caused by endolymphatic hydrops? Otol Neurotol. 2005;26:74-81.

8. Nakashima $T$, et al. Visualization of endolymphatic hydrops in patients with Meniere's disease. Laryngoscope. 2007;117:415-20.

9. Lee KJ, Kirsch CFE, Lai C, Ishiyama A. Endolymphatic sac tumor presenting with Meniere's disease. Otolaryngol Head Neck Surg. 2009;142:915-6.

10. Choi SJ, et al. A posterior petrous meningioma with recurrent vertigo. Clin Exp Otorhinolaryngol. 2012;5:234-6.

11. Mahmud MR, Khan AM, Nadol JB Jr. Histopathology of the inner ear in unoperated acoustic neuroma. Ann Otol Rhinol Laryngol. 2003;112:979-86.

12. Murata J, Horii A, Tamura M, Mitani K, Mizuki M, Kubu T. Endolymphatic hydrops as a cause of audio-vestibular manifestations in relapsing polychondritis. Acta Otolaryngol. 2006;126: 548-52.

13. Committee on Hearing and Equilibrium. Committee on Hearing and Equilibrium guidelines for the diagnosis and evaluation of 
therapy in Meniere's disease. Otolaryngol Head Neck Surg. 1995; 13:181-5.

14. Seo YJ, Kim J, Choi JY, Lee WS. Visualization of endolymphatic hydrops and correlation with audio-vestibular functional testing in patients with definite Meniere's disease. Auris Nasus Larynx. 2013;40:167-72.

15. Barath K, Schuknecht A, Monge Naldi B, Schrepfer T, Bockisch CJ, Hegemann SC. Detection and grading of endolymphatic hydrops in Meniere disease. AJNR Am J Neuroradiol. 2014;35: 1-5 epub ahead of print.

16. Ferraro JA, Durrant JD. Electrocohleography in the evaluation of patients with Meniere's disease/endolymphatic hydrops. J Am Acad Audiol. 2006;17:45-68.

17. Kimura RS, Schuknecht HF. Membranous hydrops in the inner ear of the guinea pig after obliteration of the endolymphatic sac. Pract Otolaryngol. 1965;27:343-54.

18. Kimura RS. Experimental blockage of the endolymphatic duct and sac and its effect on the inner ear of the guinea pig. Ann OtoRhino-laryng. 1967;76:664-87.

19. Andrews JC, Bohmer A. The surgical approach to the endolymphatic sac and the cochlear aqueduct in the guinea pig. Am J Otolaryngol. 1989;10:61-6.

20. Megerian CA, et al. Surgical induction of endolymphatic hydrops by obliteration of the endolymphatic duct. J Vis Exp. 2010;35:e1728.

21. Horner KC. Auditory and vestibular function in experimental hydrops. Otolaryngol Head Neck Surg. 1995;112:84-9.

22. Albers FW, De Groot JC, Veldman JE, et al. Ultrastructure of the organ of Corti in experimental hydrops. Acta Otolaryngol. 1988;105:281-91.

23. Horner KC, Cazlas Y. Rapidly fluctuating thresholds at the onset of experimentally-induced hydrops in the guinea pig. Hear Res. 1987;26:319-25.

24. Bixenstein PJ, Maniglia MP, Vasanji A, Alagramam KN, Megerian CA. Spiral ganglion degeneration patterns in endolymphatic hydrops. Laryngoscope. 2008;118:1217-23.

25. Momin SR, Melki SJ, Alagramam KN, Megerian CA. Spiral ganglion loss outpaces inner hair cell loss in endolymphatic hydrops. Laryngoscope. 2010;120:159-65.

26. Megerian CA. Diameter of the cochlear nerve in endolymphatic hydrops: implications for the etiology of hearing loss in Ménière's disease. Laryngoscope. 2005;115:1525.

27. Fraysse BG, Alonso A, House WF. Ménière's disease and endolymphatic hydrops: clinical-histopathological correlation. Ann Otol Rhinol Laryngol. 1980;89(Suppl76):2-22.

28. Hott ME, Graham M, Bonassar LJ, Megerian CA. Correlation between hearing loss and scala media area in guinea pigs with long-standing endolymphatic hydrops. Otol Neurotol. 2003;24: 64-72.

29. Salt AN, Plontke SK. Endolymphatic hydrops: pathophysiology and experimental models. Otolaryngol Clin North Am. 2010;43: 971-83.

30. Francis F, et al. A gene (PEX) with homologies to endopeptidases is mutated in patients with $\mathrm{X}$-linked hypophosphatemic rickets. Nat Genet. 1995;11:130-6.

31. Strom TM, et al. Pex gene deletions in Gy and Hyp mice provide models for X-linked hypophosphatemia. Hum Mol Genet. 1997;6:165-71.

32. Liu S, Guo R, Quarles LD. Cloning and characterization of the proximal murine Phex promoter. Endocrinology. 2001;142: 3987-95.

33. Jonsson $\mathrm{KB}$, et al. Fibroblast growth factor 23 in oncogenic osteomalacia and X-linked hypophosphatemic rickets. N Engl J Med. 2003;348:1656-63.

34. Rowe P. The chicken of the egg: PHEX, FGF23, and SIBLINGs unscrampled. Cell Biochem Funct. 2012;30:355-75.
35. Lyon MF, Scriver CR, Baker LRI, Tenenhouse HS, Kronick J, Mandla S. The Gy mutation: another cause of X-linked hypophosphatemia in mouse. Proc Natl Acad Sci. 1986;83:4899-903.

36. Weir N. Sensorineural deafness associated with recessive hypophosphataemic rickets. J Laryngol Otol. 1977;91:717-22.

37. Davies M, Kane R, Valentine J. Impaired hearing in X-linked hypophosphatemic (vitamin D-resistant) osteomalacia. Ann Intern Med. 1984;100:230-2.

38. Boneh A, Reade TM, Scriver CR, Rishikof E. Audiometric evidence for two forms of X-linked hypophosphatemia in humans, apparent counterparts of Hyp and Gy mutations in humans. Am J Med Genet. 1987;27:997-1003.

39. O'Malley S, Ramsden RT, Latif A, Kane R, Davies M. Electrocochleographic changes in the hearing loss associated with X-linked hypophosphataemic osteomalacia. Acta Otolaryngol. 1985;100:13-8.

40. O'Malley SP, Adams JE, Davies M, Ramsden RT. The petrous temporal bone and deafness in X-linked hypophosphataemic osteomalacia. Clin Radiol. 1988;39:528-30.

41. Fishman G, Miller-Hansen D, Jacobsen C, Singhal VK, Alon US. Hearing impairment in familial X-linked hypophosphatemic rickets. Eur J Pediatr. 2004;163:622-3.

42. Lorenz-Depiereux B, Guido VE, Johnson KR, Zheng QY, Gagnon LH, Bauschatz JD, Davisson MT, Washburn LL, Donahue LR, Strom TM, Eicher EM. New intragenic deletions in the Phex gene clarify X-linked hypophosphatemia-related abnormalities in mice. Mamm Genome. 2004;15:151-61.

43. • Megerian CA, Semaan MT, Aftab S, Kisley LB, Zheng QY, Pawlowski KS, Wright CG, Alagramam KN. A mouse model with postnatal endolymphatic hydrops and hearing loss. Hear Res. 2008;237:90-105. This article is the first to describe the otolgic manifestations in the Phex Hyp-Duk/Y murine model. The postnatal time course of vestibular dysfunction and hearing loss are correlated with histologic evidence of progressive endolymphatic hydrops. This study also documents patency of the endolymphatic duct, thus making this model of endolymphatic hydrops different than the time and cost-intensive surgical guinea pig model.

44. Nadol JB Jr, Thornton AR. Ultrastructural findings in a case of Meniere's disease. Ann Otol Rhinol Laryngol. 1987;96:449-54.

45. Nadol JB Jr. Degeneration of cochlear neurons as seen in the spiral ganglion of man. Hear Res. 1990;49:141-54.

46. Nadol JB Jr, Adams JC, Kim JR. Degenerative changes in the organ of Corti and lateral cochlear wall in experimental endolymphatic hydrops and human Meniere's disease. Acta Otolaryngol. 1995;519:47-59.

47. •• Semaan MT, Zheng QY, Han F, Yu H, Heaphy JC, Megerian CA. Characterization of neuronal cell death in the spiral ganglia of a mouse model with endolymphatic hydrops. Otol Neurotol 2013;34:559-569. This article demonstrates the critical role of caspase and the apoptotic cascade in spiral ganglion neuron (SGN) degeneration caused by endolymphatic hydrops. It quantifies the apical to basal topographic pattern of SGN loss that has been observed in variety of animal and human endolymphatic hydrop conditions. The article also suggests future research and therapeutic targets for Meniere's disease.

48. Lamkanfi M, Declercq W, Kalai M, Saelens X, Vandenabeele P. Alice in caspase land: a phylogenetic analysis of caspases from worm to man. Cell Death Differ. 2002;9:358-61.

49. Warnecke A, Wissel K, Hoffmann A, Hoffman N, Berkingali N, Gro G, Lenarz T, Stover T. The biological effects of cell-delivered brain-derived neurotrophic factor on cultured spiral ganglion cells. NeuroReport. 2007;18:1683-6.

50. - Semaan MT, Megerian CA. Meniere's disease: a challenging and relentless disorder. Otolaryngol Clin N Am 2011;44:383-403. This article presents a clinical vignette of a patient with 
Meniere's disease (MD) who ultimately displays the full spectrum of the disorder. The subsequent review provides an up-to-date, comprehensive review of the clinical, diagnostic, and therapeutic elements of $M D$.

51. Kim HH, Wiet RJ, Battista RA. Trends in the diagnosis and the management of Meniere's disease: results of a survey. Otolaryngol Head Neck Surg. 2005;132:722.

52. Ruben R, Sekula J, Bordely JE. Human cochlear responses to sound stimuli. Ann Otorhinolaryngol. 1960;69:459-76.

53. Schmidt PH, Eggermont JJ, Odenthal DW. Study of Menière's disease by electrocochleography. Acta Otolaryngol. 1974;316:75-84.

54. Ferraro JA, Durrant JD. Electrocochleography. In: Katz J, editor. Handbook of clinical audiology. New York: Lippincott, Williams and Williams; 2002. p. 249-73.

55. Ferraro JA. Electrocochleography: a review of recording approaches, clinical applications, and the new findings in adults and children. J Am Acad Audiol. 2010;21:145-52.

56. Gatland DJ, Billings RJ, Youngs RP, et al. Investigation of the physiological basis of summating potential changes in endolymphatic hydrops. Acta Otolaryngol. 1988;105:218.

57. Ferraro JA, Arenberg IK, Hassanein RS. Electrocochleography and symptoms of inner ear dysfunction. Arch Otolaryngol. 1985;111:71-4.

58. Ge X, Shea JJ. Transtympanic electrocochleography: a 10-year experience. Otol Neurotol. 2002;23:799-805.

59. Van Deelen GW, Ruding PR, Veldman JE, et al. Electrocochleographic study of experimentally induced endolymphatic hydrops. Arch Otorhinolaryngol. 1987;244:167-73.

60. - Melki SJ, Li Y, Semaan MT, Zheng QY, Megerian CA, Alagramam KN. A mouse model validates the utility of electrocochleography in verifying endolymphatic hydrops. J Assoc Res Otolaryngol 2014 [epub ahead of print; doi: 10.1007/s10162-0140445-0]. This study is the first description of an in vivo correlation between ECochG and spontaneous ELH. Melki et al. showed that an elevated SP/AP ratio predicted presence of ELH, although not necessarily the severity of ELH. This study exemplifies the unique research potential of the PhexHyp-Duk/Y model for real-time histopathologic correlations.
61. Rauch SD, Zhou G, Kujawa SG, et al. Vestibular evoked myogenic potentials show altered tuning in patients with Meniere's disease. Otol Neurotol. 2004;25:333-8.

62. Sheykholeslami K, Megerian CA, Zheng QY. Vestibular evoked myogenic potentials in normal mice and Phex mice with spontaneous endolymphatic hydrops. Otol Neurotol. 2009;30:535-44.

63. Semaan MT, Megerian CA. Contemporary perspectives on the pathophysiology of Meniere's disease: implications for treatment. Curr Opin Otolaryngol Head Neck Surg. 2010;18:392-8.

64. Melki SJ, Heddon CM, Frankel JK, Levitt AH, Momin SR, Alagramam KN, Megerian CA. Pharmacological protection of hearing loss in the mouse model of endolymphatic hydrops. Laryngoscope. 2010;120:1637-45.

65. Mukherjea D, et al. Transtympanic administration of short interfering (si)RNA for the NOX3 isoform of NADPH oxidase protects against cisplatin-induced hearing loss in the rat. Antioxid Redox Signal 2010;13L 589-598.

66. Rybak LP, Mukherjea D, Jajoo S, Kaur T, Ramkumar V. siRNAmediated knock-down of NOX3: therapy for hearing loss? Cell Mol Life Sci. 2012;69:2429-34.

67. Ahmed Z, et al. Ocular neuroprotection by siRNA targeting caspase-2. Cell Death Dis. 2011;2:e173.

68. Carpenter TO, Imel EA, Holm IA. Jan de Beur SM, Insogna KL. A clinician's guide to X-linked hypophosphatemia. J Bone Miner Res. 2011;26:1381-8.

69. Verge CF, et al. Effects of therapy in X-linked hypophosphatemic rickets. N Engl J Med. 1991;325:1843.

70. Imel EA, DeMeglio LA, Hui SL, Carpenter TO, Econs MJ. Treatment of X-linked hypophosphatemia with calcitriol and phosphate increases circulating fibroblast growth factor 23 concentrations. J Clin Endocrinol Metab. 2010;95:1846-50.

71. AonoY, et al. Anti-FGF-23 neutralizing antibodies ameliorate muscle weakness and decreased spontaneous movement of Hyp mice. J Bone Miner Res 2011;26:803-810. 